\title{
Experience With Capstone Projects BASED ON COLDFIRE MICROCONTROLLERS
}

\author{
Naraig Manjikian \\ Department of Electrical and Computer Engineering, Queen's University, Kingston, Ontario, Canada K7L 3N6 \\ naraig.manjikian@queensu.ca
}

\begin{abstract}
This paper describes the experience of the author in supervising capstone undergraduate projects that have used microcontroller chips based on the ColdFire processor architecture, along with supporting hardware and software. Six capstone design projects are selected for illustration, and these projects have been pursued by a total of nineteen students under the supervision of the author in the Department of Electrical and Computer Engineering at Queen's University between 2013 and 2017. After summarizing the selected projects to provide the context, this paper highlights the supervisory role of the author to provide potentially useful insights to other potential project supervisors. A retrospective assessment is provided for the decision to use ColdFire-based platforms in these projects, along with some reflections on the experience. Considerations for platform selection by the author in future projects are also outlined.
\end{abstract}

Keywords: Capstone projects, supervisory role, commercial microcontrollers, software development tools, operating systems software, embedded systems, robotic applications.

\section{INTRODUCTION}

This paper describes the experience of the author serving as the supervisor of capstone projects for undergraduate students in the Department of Electrical and Computer Engineering at Queen's University where the projects have involved the use of ColdFire microcontroller chips, along with supporting hardware and software. These have been supervisor-proposed projects, but students have also introduced their own facets. For the benefit of other potential supervisors considering various products to use in comparable capstone projects, this paper highlights the role of the author in supervising ColdFire-based projects, provides an assessment with advantages and disadvantages of the choice of ColdFire products, and discusses various considerations for the selection of platforms by the author for future capstone projects.
For consideration in this paper, the author has selected six ColdFire-based capstone projects pursued by a total of nineteen students. These projects were pursued under the supervision of the author over the period 2013-2017. All of the projects have involved interfacing with sensors and motors, with five of the six projects specifically involving mobile wheeled robots.

Sensors for most of the projects have included infrared proximity devices and three-axis accelerometers connected to on-chip analog-to-digital converters. One of the projects used the Microsoft Kinect sensor connected to a laptop computer with processed data then sent to an embedded platform.

Three types of actuators have been used across the collection of projects: standard servomotors, continuousrotation servomotors, and DC motors. Direct pulse-widthmodulated (PWM) output has controlled servomotors, whereas a PWM connection to a separate motor control board has indirectly managed DC motors. ColdFire chips (and a number of similar products from other vendors) can include PWM interfaces as a convenience for embeddedsystems design related to motor-control applications.

The remainder of this paper provides an overview of ColdFire-based hardware products and relevant software support, describes projects originally proposed by the author that have been pursued by students with the intent to use ColdFire-based platforms, offers insight for other potential supervisors of similar capstone projects through reflections on the experience with the selected projects, and provides final comments in a concluding section.

\section{COLDFIRE PRODUCTS AND VENDOR SUPPORT}

This section provides an overview of the ColdFire architecture, the range of products that incorporate implementations of the ColdFire architecture, and the various hardware and software offerings from the vendor to support applications based on ColdFire.

The 32-bit ColdFire processor architecture [1] was originally developed by Motorola before it separated its microelectronics business into Freescale Semiconductor, 
which was then acquired by NXP. ColdFire-based microcontroller chips with varying levels of sophistication incorporate Flash EEPROM, RAM, and various input/output interfaces.

For the projects considered in this paper, two particular ColdFire chip variants are relevant. At the upper end of the scale of sophistication is the ColdFire MCF54418 [2] that incorporates hardware support for analog-digital conversion, USB, SPI, CAN, Ethernet, and numerous other interfaces, along with an on-chip controller for external synchronous DRAM memory. A full version of the Linux operating system can be supported on this product. A less sophisticated product is the ColdFire MCF52259 [3] with no support for external DRAM memory, relying primarily on on-chip Flash EEPROM and SRAM, which precludes the use of larger software such as Linux. The MCF52259 does not offer the same variety of interfaces as the more sophisticated chip, but it still includes Ethernet support.

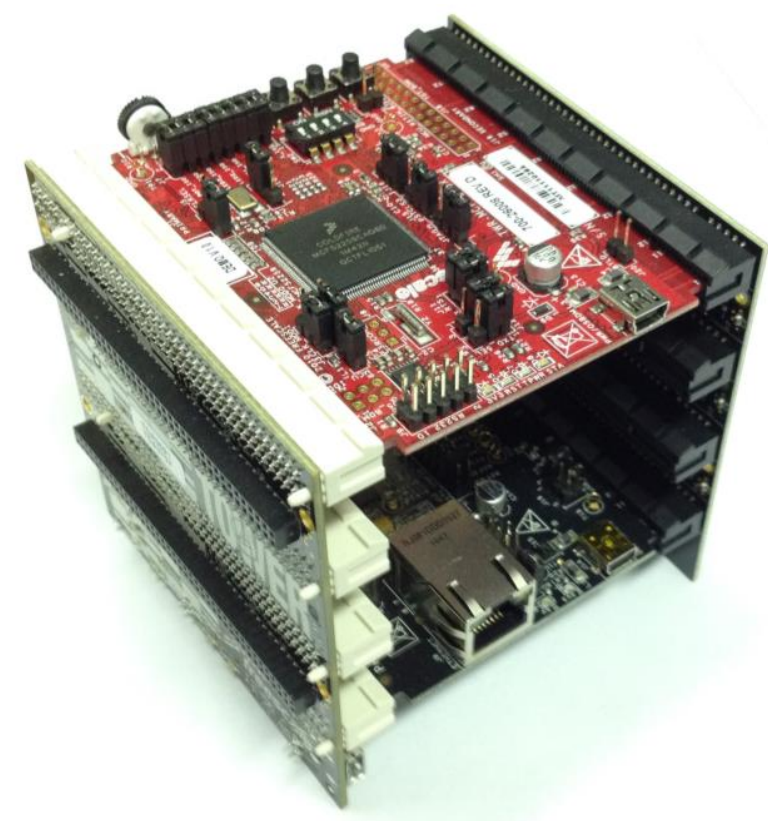

Fig. 1. Tower with MCF52259 board and Ethernet board.

The vendor of ColdFire chips has sought to encourage commercial adoption by producing and marketing ColdFire development/evaluation boards. Although standalone evaluation boards have been offered, the vendor has also developed the more modular Tower environment [4] within which various Tower-compatible microcontroller and peripheral boards can be used. Figure 1 shows a Tower configuration with a MCF52259 microcontroller module [5] and a communication module with an Ethernet jack [6] (the hardware support for Ethernet is inside the MCF52259 chip). The vendor had developed a Tower module with the MCF54418, but as will be discussed later in the paper, discontinuation of that module has presented challenges for project activity.

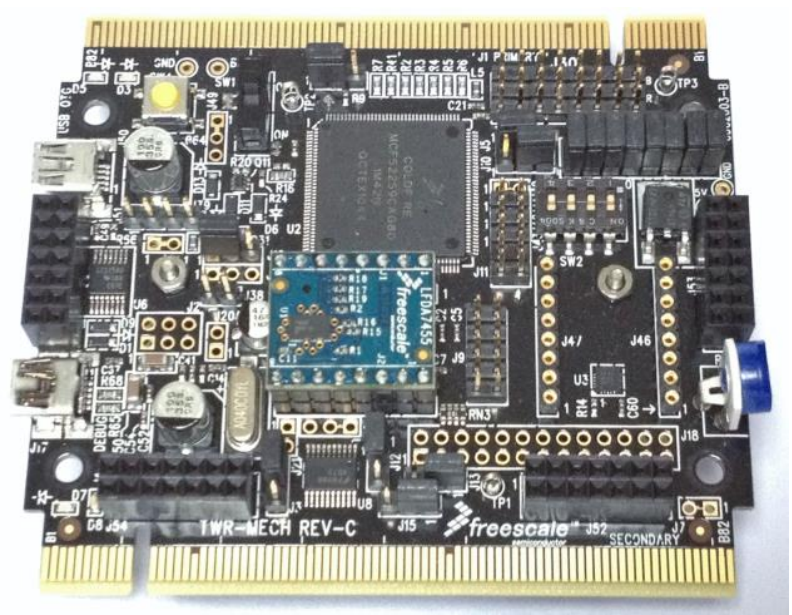

Fig. 2. MCF52259-based mechatronics board for Tower with eight servomotor headers at top-right corner.

Another Tower-compatible development product of particular interest to the subject of this paper is shown in Fig. 2. It is a mechatronics-oriented MCF52259 module with convenient headers for direct connection of standard servomotors [7]. On the back of this module is a battery pack for four AA-size batteries. This board can be used on its own, or it can be combined with the Ethernet board shown in Fig. 1 within a Tower for network-capable motor-control applications.

In terms of software, the vendor has provided no-fee evaluation versions of application development tools to encourage adoption. Of particular interest for the efforts considered in this paper is the older version 7 of the CodeWarrior tool [8], for which a no-fee limited-codesize variant is available and quite adequate for the type of activity pursued in capstone projects.

Finally, the vendor has provided the no-fee but licensed MQX real-time embedded operating system software [9] as another product to encourage adoption of ColdFire hardware. Version 3 of MQX is of particular interest for the subject of this paper because it is compatible with the older version 7 of CodeWarrior for application development, and it can be supported by the less sophisticated MCF52259 chip.

\section{CAPSTONE PROJECTS USING COLDFIRE}

This section first briefly summarizes the administration of capstone projects in the Department of Electrical and Computer Engineering at Queen's University. Then, six selected projects from the period 2013 to 2017 are described. The intent of each one was to produce a ColdFire-based embedded-system implementation. Specific challenges encountered with respect to the choice of 
ColdFire are outlined, along with an indication of the eventual outcome in terms of implementation.

Activity for capstone projects begins during the winter term of third year in a project-preparation course where students can choose a supervisor-proposed project or seek to identify a supervisor willing to oversee a studentproposed project. An initial proposal document is submitted at the end of the Winter term. Then, in a full-year project course during the Fall and Winter terms of the fourth year, students first submit a more detailed technical proposal document, then proceed with design and implementation activity with a $\$ 400$ budget. Completed projects are demonstrated at an annual open-house event in the Winter term. At the end, students submit a final report. The academic sessions for each instance of full-year project activity are indicated in the following paragraphs, along with the project descriptions and student names.

2013-2014 Wheeled Robotic Project: The author proposed the development of mobile autonomous wheeled robots. With recent experience from an industrial collaboration [10] that involved the use of the Linux operating system on a sophisticated ColdFire MCF54418 chip, the author proposed that students interested in this project use a MCF54418-based Tower kit. The use of Linux and the hardware Ethernet interface on the MCF54418 chip would enable network capability for this project. Linux drivers for PWM output and analog-digital conversion were available for interfacing with motors and sensors.

A first group consisting of students D. Ipema, A. Sanghi, and Y. Zang selected this project and pursued the development of a physically larger robot platform with more powerful DC motors and a more substantial battery. The students proposed an extension involving the use of the Microsoft Kinect for a vision sensor connected to a laptop which would then send processed data or commands to the MCF54418 chip that controlled the motors. All of this equipment would be mounted on the wheeled platform. The group made initial progress in configuring and testing the Tower kit with Linux. Unfortunately, in the course of hardware testing near the end of the Fall 2013 term, the MCF54418 circuit board was damaged through some type of electrical fault and would not operate at all. Even more unfortunately, Freescale had discontinued production of the MCF54418 board, and distributors no longer had it in stock. As a result, the students migrated to an Arduino platform at the beginning of Winter 2014 for the microcontroller portion of the project so that they could ultimately produce a working system by the project demonstration day with object detection through the Kinect sensor causing the wheeled platform to stop moving.

A second group consisting of students T. Moussette, N. Jackson, and S. McGill also selected this project. This group opted to use a smaller prefabricated platform with less powerful DC motors and simpler infrared proximity sensors. With knowledge of the difficulty faced by the first group, this group sought to avoid a similar loss of equipment, but approximately one week before the project demonstration day in Winter 2014, the MCF54418 chip began to malfunction. Some type of hardware fault caused it to rapidly overheat soon after power-up and then cease functioning during initial software execution. With limited time before the project demonstration day, the students elected to transition rapidly to an Arduino platform to achieve the desired functionality of object detection and motion around recognized objects.

2014-2015 Wheeled Robotic Project: Because the MCF54418 using Linux was no longer feasible, the author proposed a robotic project for the following year based on the less sophisticated MCF52259 chip and the embedded MQX operating system. In addition to PWM and analogdigital conversion support, the MCF52259 chip includes a hardware Ethernet interface, and MQX provides the necessary software support. Therefore, this alternative platform would still have network capability. Furthermore, the author identified a battery-powered wireless router, the TP-LINK TL-MR3040 [11], that would easily transform a wired network connection to a wireless network connection. Finally, the author had developed a custom extension [12] for the MQX operating system to enable rapid and convenient application software development using volatile RAM within a ColdFire chip instead of having to repeatedly erase and program the non-volatile Flash EEPROM memory for every modification of application code. This extension would be provided to students.

The project was taken by a group that initially consisted of three students, but once the serious effort began on the project in September 2014, the group had been reduced to just two students, I. Little and A. ElShakankiry. This group mounted an instance of the MCF52259 Tower (as in Fig. 1) atop a prefabricated wheeled platform with DC motors which were controlled with PWM output to an intervening motor control board. In addition to demonstrating autonomous mobility for their robot, this group successfully pursued an interesting extension where a Microsoft Xbox controller connected to a laptop was used for manual directional input, with commands sent wirelessly from the laptop to the MCF52259 Tower using the battery-powered wireless router mentioned in the preceding paragraph. This group did suffer an equipment loss due to an electrical fault of some kind. This time, however, the selected MCF52259 platform had not been discontinued by the vendor, and a replacement could be readily obtained so that the project was not adversely affected.

2015-2016 Mechatronics Project: The author proposed a project on the mechatronics-oriented MCF52259 board shown in Fig. 2. This project would involve software processing the input from the on-board accelerometer in an appropriate manner to modify the PWM output to servomotors. A Tower configuration would not necessarily be used; the board in Fig. 2 can operate in a self-contained 
manner with on-board batteries to power the electronics and any attached servomotors.

A group consisting of students A. Wang, M. Wightman, and R. Dutkiewicz accepted this project. Their mechatronic application involved pairs of servomotors in pan-and-tilt brackets mounted with the MCF52259 board on a flat platform. Software was developed to maintain the end-effectors of the pan-and-tilt arrangements in a fixed orientation by reading the on-board accelerometer, performing numerical calculations to determine the appropriate PWM outputs for the tilt angles in two dimensions, and controlling the servomotors accordingly. The students elected to use the later version 10 of the CodeWarrior development software; comments regarding this version will be provided later in this paper.

2015-2016 Parallel-Parking Robot Project: The author proposed a project to use the mechatronics-oriented MCF52259 board shown in Fig. 2 along with continuousrotation servomotors and appropriate sensor hardware to reflect the trend of computer-assisted - if not fully automated - parallel parking in full-size automobiles. The author sought to have wireless networking capability for this type of project, hence the use of a Tower with the Ethernet communication board was deemed necessary. By this time, a smaller battery-powered wireless router from IOGEAR [13] had been identified for the purpose of providing wireless connectivity.

A group consisting of students J. Fan, T. Hsieh, M. Lapinsky, and S. Knowles pursued this project. They constructed their own wood-based platform on which to mount continuous-rotation servomotors for driving the wheels, a Tower with mechatronics-oriented MCF52259 board and Ethernet board, and a single infrared proximity sensor. They developed software to use that sensor to detect an empty space of sufficient size on the side of the wheeled robot as it drove straight, then maneuver the robot in reverse with appropriate turns to park in the available space. Their development efforts were expedited with the custom extension for MQX by the author to support RAM-based application code [12]. They were also able to use the IOGEAR wireless router for network connectivity.

2016-2017 Parallel-Parking Robot Project: Another group consisting of students D. Marcuccio, D. Suh, K. Y. Ho, and M. Keyes pursued a similar parallel-parking project in the following year, reusing the same physical platform and Tower hardware as the previous group. The second group pursued their own solution that was somewhat more sophisticated by having three infrared proximity sensors, with one mounted pointing to the rear at a 45degree angle for sensing the orientation of the platform during the parking maneuver. This group also benefitted from the custom MQX extension developed by the author, and the wireless router was also utilized for network connectivity.

\section{ROLE OF THE SUPERVISOR}

Having described ColdFire-related hardware and software, and having summarized the selected projects, it is now meaningful to outline the role of the author as supervisor for those projects in the context of the technical issues and challenges arising from the choice of ColdFire.

A general comment can be made initially that any commercial microcontroller product has a certain level of complexity that is reflected, for example, in the size of reference documentation, which can easily exceed several hundred pages [2], [3]. If such a product is used in any relevant course(s) prior to a capstone project, there can be an advantage for students. If that is not the case and if the supervisor is not knowledgeable about the chosen platform, there can be some potential disadvantages.

In the case of ColdFire-based microcontrollers, the author has acquired considerable experience, including knowledge of important subtleties in the documentation. In particular, the complexities of configuring the various hardware interfaces for ColdFire chips involves the use of important information in disparate locations of the relevant documents. Considerable time can be lost with nonfunctioning hardware if even a single obscure aspect is overlooked or improperly configured. Students seeking to be employed in technical work should certainly experience the realities of technical documentation and complex hardware from commercial vendors, but not at the expense of severe progress-limiting delays that would not be experienced by students pursuing other types of projects without comparable challenges. Therefore, the author has provided appropriate guidance to enable students to progress reasonably, while still requiring them to truly make their own efforts to develop relevant software for using the interfaces provided in a ColdFire chip. If students have not progressed after demonstrating that they have invested a sufficient effort on their own, then the author has provided more direct assistance to guide them towards a solution.

A further role for a supervisor is to provide specialized software that can be valuable for microcontroller-based projects, either to aid the development effort or to be incorporated into the final application. Providing software in this manner is certainly subject to the requirement that the students working on a project still contribute either a substantial amount of code themselves or a sufficiently non-trival solution that demonstrates good insight. One example of this role that was mentioned previously is the custom extension for MQX that the author developed to use on-chip RAM for application code so that Flash EEPROM would not require frequent erasure and reprogramming. Another example not mentioned previously is simply directing students to vendor-provided sample code that can be adapted or extended to suit the needs of a particular project, and again there is the expectation that stu- 
dents contribute of something of appropriate quantity or quality beyond the starting point of sample code.

Finally, for any projects where circuitry is more exposed or manipulated, the author as supervisor has always attempted to instill a strong degree of caution in students to avoid potential damage to electronic components. By using anecdotes of direct experience related to actual failures with exploding chips, the author has sought to encourage students to ensure, for example, that all power connections are verified before systems are activated, and that care is taken to prevent short-circuits. Nonetheless, three of the six projects described in this paper still suffered equipment loss due to various electrical issues.

\section{REFLECTIONS ON EXPERIENCE AND CONSIDERATIONS FOR THE FUTURE}

After several years of supervising ColdFire-based projects, the author can meaningfully reflect on the experience. As with all technical activity, any choices that are made have various advantages and disadvantages to, with some being understood at the beginning, and others emerging through experience.

In the case of ColdFire-based hardware platforms, the experience with the Tower system has generally been positive (putting aside the MCF54418 module discontinuation by the vendor that affected two of the six projects). As long as appropriate care was employed, the Tower platform was reasonably robust from a physical standpoint. The reality of potential electrical damage has already been addressed earlier in this paper; vigilance and attention to detail may not necessarily eliminate the likelihood of such damage.

Projects benefitted from the availability of network connectivity provided through the Ethernet module in the Tower. For the three projects that utilized the mechatronics-oriented MCF52259 module, the built-in battery pack and headers for servomotor connections were quite convenient.

The on-chip Flash EEPROM and RAM of the MCF52259 are sufficient to support the MQX embedded operating system with networking enabled, along with application code, hence it is certainly possible to pursue projects for non-trivial embedded applications.

With the appropriate initial guidance on navigating the voluminous documentation (e.g., indications of which chapters or sections to read carefully), students were generally able to address the complexity. In a few instances, the author as supervisor had to provide more direct guidance.

In terms of the software aspects, the no-fee CodeWarrior software version that can generate MQX-based code without exceeding the code-size limitation is an older software tool that is certainly still usable. It does, howev- er, have one important drawback in that it requires using a 32-bit version of the Microsoft Windows operating system. Newer desktop computers and laptop computers have 64-bit versions. Fortunately, smaller tablet computers often use 32-bit versions of Microsoft Windows, and the author has successfully used the older CodeWarrior software on versions 7, 8, and 10 of 32-bit Microsoft Windows. A later version 10 of CodeWarrior is compatible with 64-bit Windows, but its drawback is that newer MQX software must also be used, and that MQX version is large enough to exceed the no-fee code-size limitation. One of the projects discussed in this paper used CodeWarrior version 10 because MQX was not part of that project.

Finally, for MQX itself, there is certainly complexity involved because it is a full operating system albeit for embedded purposes. Through a combination of sample code from the vendor, further developed template code from the author as supervisor, and the ability of students to navigate the MQX documentation, it was generally the case that this complexity could be reasonably addressed.

Future Considerations: Although most of the projects discussed in this paper produced a functioning ColdFirebased system, and although products such as the mechatronics-oriented MCF52259 board can be suitable platforms for projects, there are some considerations that would guide future platform selections toward other options.

First, the ColdFire architecture is not the most popular one for embedded products. With the corporate changes that have occurred for the vendor, the availability of some or many ColdFire-based products might change, as exemplified by the discontinuation of the MCF54418 board.

Second, the courses on digital logic, computer organization, and embedded systems in the Department of Electrical and Computer Engineering at Queen's University migrated several years ago to using platforms and intellectual property based on programmable logic chips from Altera [14], [15], [16] rather than fixed microcontroller chips. Emphasizing the use of programmable logic chips in capstone projects for embedded applications would offer advantages related to familiarity while still having industrial-calibre sophistication.

Third, the need for 32-bit Microsoft Windows to support the older CodeWarrior software so that MQX code can be generated to fit within the code-size limitations of the no-fee version is a potentially significant limitation for the future. With the later 64-bit-compatible CodeWarrior software, MQX code cannot be generated with the no-fee version. The author has some experience with Towercompatible ARM-based modules and using the 64-bitcompatible no-fee CodeWarrior to successfully generate MQX code for ARM within the code-size limitation, but there has been no student project experience.

For these reasons, the author has made a decision to give priority to the selection of programmable-logic-based 
platforms for future student projects over the next few years. Programmable-logic boards intended for batterypowered operation are available, and analog-digital capability can be provided through a connection between a logic chip and an analog conversion chip [17]. It is possible to control servomotors directly from a programmable logic chip; the author has experimented with the hardware and software support to achieve this functionality.

\section{CONCLUSION}

This paper has described the experience and perspective of the author as supervisor for six capstone projects involving the use of ColdFire-based microcontroller chips for wheeled robots or other motor-based applications. After providing a summary of hardware and software products related to the ColdFire architecture and after describing the selected student projects, the supervisory role of the author for these projects was outlined and reflections were provided on the experience with these projects, all for the benefit of other potential supervisors contemplating similar projects. Generally positive outcomes with functioning systems involving interesting capabilities such as wireless networking capability provide a favorable perspective on the use of ColdFire-based products.

There are, however, certain considerations related to hardware availability, software development tools, and embedded operating system software for ColdFire that could become more significant issues for future projects. Based on those considerations and others, the author intends to emphasize the use of platforms based on programmable logic chips that would be more familiar to students based on their exposure to programmable logic in earlier courses.

\section{References}

[1] Freescale Semiconductor, Inc., ColdFire Family Programmer's Reference Manual, Document CFPRM Rev. 3, March 2005.

[2] Freescale Semiconductor, MCF5441x Reference Manual, 2010.

[3] Freescale Semiconductor, Inc., MCF52259 ColdFire Integrated Microcontroller Reference Manual, Document MCF52259RM Rev. 4, March 2011.

[4] Freescale Semiconductor Inc., Freescale Tower System Development Board Platform, Document TWRFS Rev. 17, 2014.

[5] Freescale Semiconductor, Inc., TWR-MCF5225X User Manual, Rev. 1, September 2009.
[6] Freescale Semiconductor, Inc., User Manual for TWR-SER, Document TWRSERUM, Rev. 0, May 2009.

[7] Freescale Semiconductor, Inc., Tower System Mechatronics Board and Robot - Fact Sheet, Document TWRMECHFS Rev. 1, 2012.

[8] Freescale Semiconductor, Inc., Freescale MQX RTOS Reference Manual, Document MQXRM Rev. 2, September 2009.

[9] Freescale Semiconductor, Inc., CodeWarrior Development Studio for ColdFire Architectures V7.2, Document 95000011 Rev. H, 2010.

[10] N. Manjikian, L. Katona, A. Ferdynus, S. V. Chamlian, and P. Katkov, "Implementation and performance assessment of Linux device drivers for the ColdFire MCF54418 microcontroller," Proceedings of the 2013 Canadian Conference on Electrical and Computer Engineering, Regina, Saskatchewan, May 2013.

[11] TP-LINK Technologies Co., Ltd., TL-MR3040 Portable 3G/3.75G Battery Powered Wireless N Router - User Guide, Document 1910010615 rev 1.0.0, 2012.

[12] N. Manjikian, "Dynamic RAM-based programs and tasks in the Freescale MQX Operating System," Proceedings of the 2015 Canadian Conference on Electrical and Computer Engineering, Halifax, Nova Scotia, May 3-6, 2015, pp. 706711 .

[13] IOGEAR, NetShair Nano Ultra Portable Wi-Fi Router/Access Point - Data Sheet, 2015.

[14] Terasic Inc., DEO User Manual, document version 1.6, May 2012.

[15] Altera Corp., Altera Monitor Program Tutorial, document version for Quartus II 13.0, May 2013.

[16] Altera Corp., Basic Computer System for the Altera DEO Board, document version for Quartus II 13.0, May 2013.

[17] Terasic Inc., DEO-Nano User Manual, document version 2.0, July 2016. 